\title{
VEGF in the Crosstalk between Human Adipocytes and Smooth Muscle Cells: Depot-Specific Release from Visceral and Perivascular Adipose Tissue
}

\author{
Raphaela Schlich, ${ }^{1}$ Miriam Willems, ${ }^{1,2}$ Sabrina Greulich, ${ }^{3}$ Florian Ruppe, ${ }^{2}$ \\ Wolfram Trudo Knoefel, ${ }^{2}$ D. Margriet Ouwens, ${ }^{3,4}$ Bujar Maxhera, ${ }^{5}$ Artur Lichtenberg, ${ }^{5}$ \\ Jürgen Eckel, ${ }^{1}$ and Henrike Sell ${ }^{1}$ \\ ${ }^{1}$ Paul-Langerhans-Group, Integrative Physiology, German Diabetes Center, Auf'm Hennekamp 65, 40225 Düsseldorf, Germany \\ ${ }^{2}$ Department of General-, Visceral-, and Pediatric Surgery, Heinrich-Heine-University and University Hospital Düsseldorf, \\ 40225 Düsseldorf, Germany \\ ${ }^{3}$ Signal Transduction, Institute of Clinical Biochemistry and Pathobiochemistry, German Diabetes Center, 40225 Düsseldorf, Germany \\ ${ }^{4}$ Department of Endocrinology, Ghent University Hospital, 9000 Ghent, Belgium \\ ${ }^{5}$ Department of Cardiovascular Surgery, Düsseldorf University Hospital, 40225 Düsseldorf, Germany
}

Correspondence should be addressed to Henrike Sell; henrike.sell@ddz.uni-duesseldorf.de

Received 28 February 2013; Revised 10 June 2013; Accepted 10 June 2013

Academic Editor: Daniel Konrad

\begin{abstract}
Copyright (C) 2013 Raphaela Schlich et al. This is an open access article distributed under the Creative Commons Attribution License, which permits unrestricted use, distribution, and reproduction in any medium, provided the original work is properly cited.

Adipose tissue secrets adipokines and fatty acids, which may contribute to obesity-associated vascular dysfunction and cardiovascular risk. This study investigated which factors are responsible for the synergistic effect of adipokine and oleic acid(OA-) induced proliferation of human vascular smooth muscle cells (VSMC). Adipocyte-conditioned medium (CM) from human adipocytes induces proliferation of VSMC in correlation to its vascular endothelial growth factor (VEGF) content. CM increases VEGF-receptor (VEGF-R) 1 and 2 expression and VEGF secretion of VSMC, while OA only stimulates VEGF secretion. VEGF neutralization abrogates CM- and OA-induced proliferation and considerably reduces proliferation induced by $\mathrm{CM}$ and $\mathrm{OA}$ in combination. VEGF release is higher from visceral adipose tissue (VAT) of obese subjects compared to subcutaneous adipose tissue (SAT) and VAT from lean controls. Furthermore, VEGF release from VAT correlates with its proliferative effect. Perivascular adipose tissue (PAT) from type 2 diabetic patients releases significantly higher amounts of VEGF and induces stronger proliferation of VSMC as compared to SAT and SAT/PAT of nondiabetics. In conclusion, VEGF is mediating CM-induced proliferation of VSMC. As this adipokine is released in high amounts from VAT of obese patients and PAT of diabetic patients, VEGF might link adipose tissue inflammation to increased VSMC proliferation.
\end{abstract}

\section{Introduction}

Obesity has become a major worldwide health problem, especially in industrial countries, and is associated with a number of metabolic diseases and secondary complications, including insulin resistance, type 2 diabetes, atherosclerosis, and cardiovascular disease [1]. It is well established that adipose tissue is an endocrine organ releasing lipid mediators and a variety of proteins, the so-called adipokines [2]. Increasing evidence indicates that obesity is causally linked to a chronic low-grade systemic inflammatory state $[3,4]$ that contributes to obesity-associated vascular dysfunction and cardiovascular risk [5]. Obesity is strongly related to the development of atherosclerosis in humans as well as in various animal models [6,7]. In this context, visceral obesity confers the highest risk for the development of metabolic and cardiovascular diseases [8]. Specifically in the pathophysiology of vascular diseases, perivascular adipose tissue might play an important role because almost all blood vessels are surrounded by this fat depot, and due to the fact 
that perivascular adipocytes are not separated from the blood vessel wall by an anatomic barrier, the secretion of adipokines by this fat depot may provide a new link between obesity and vascular complications [9]. However, till now the mechanism how visceral and perivascular fat enhances the risk of metabolic and cardiovascular disease is not completely unraveled. Besides endothelial cells, vascular smooth muscle cells (VSMC) represent one of the major cell types of the vascular wall retaining homeostasis of the vessel wall. Arterial wall thickening is mediated by migration of VSMC from the media to intima and their concomitant proliferation. We previously established a model of adipocyte-conditioned medium (CM) inducing VSMC proliferation [10]. The combination of $\mathrm{CM}$ with oleic acid (OA) increased the proliferation in a synergistic way via induction of iNOS expression, NO production, and proinflammatory signaling. However, until now the mechanisms and factors, which are responsible for aberrant VSMC proliferation induced by lipid mediators and adipokines, are not fully understood. Therefore, the main objective of this study was to identify potential candidates that mediate the crosstalk between adipose tissue and VSMC potentially linking obesity and atherosclerosis. Using paired biopsies of visceral and subcutaneous adipose tissue (VAT and SAT) as well as perivascular adipose tissue (PAT) derived from lean, obese, and obese type 2 diabetic patients, this study is also aimed at relating the in vitro findings to a more physiological setting. Our study demonstrates that the vascular endothelial growth factor (VEGF) content of CM is positively correlated with CM-induced VSMC proliferation and that VEGF neutralization completely abrogates CM- and OAinduced proliferation. CM and VEGF regulate expression of VEGF-receptor (VEGF-R) 1 and 2. The secretory output from VAT and PAT of patients with type 2 diabetes and/or obesity contains high amounts of VEGF and stimulates proliferation and VEGF-R1/2 expression as compared to SAT.

\section{Materials and Methods}

2.1. Materials. Reagents for SDS-PAGE were supplied by Amersham Pharmacia Biotech (Braunschweig, Germany) and by Sigma (Munich, Germany). Polyclonal antibodies raised against VEGF-R 1 and 2 were supplied by cell signalling technology (Frankfurt, Germany), and the anti-actin antibody came from Abcam (Cambridge, Great Britain). Vascular endothelial growth factor (VEGF) neutralization was achieved by pretreatment of CM with $1 \mu \mathrm{g} / \mathrm{mL}$ VEGFneutralizing antibody from R\&D Systems (WiesbadenNordenstadt, Germany) for 1 hour. HRP-conjugated goat anti-rabbit and goat anti-mouse IgG antibodies came from Promega (Mannheim, Germany). Collagenase NB4 was obtained from Serva (Heidelberg, Germany). Troglitazone and BSA (fraction V, fatty acid free, low endotoxin) were obtained from Sigma (München, Germany). Human recombinant VEGF was purchased from Millipore GmbH (Schwalbach, Germany). The cell proliferation ELISA (BrdU, chemiluminescent) and protease inhibitor cocktail tablets were from Roche (Mannheim, Germany). VEGF ELISA kits were purchased from BioVendor GmbH (Heidelberg, Germany).
FCS was supplied by Gibco (Invitrogen, Carlsbad, USA). Sodium salt of OA (Sigma, München, Germany) was dissolved in water as a $6 \mathrm{mM}$ stock solution and was further diluted in sterile serum-free VSMC medium containing $4 \%$ (wt/v) BSA for coupling. OA was applied to VSMC at a final concentration of $100 \mu \mathrm{mol} / \mathrm{L}$ for $18 \mathrm{~h}$. All controls of experiments involving fatty acids were treated with BSA alone. All other chemicals were of the highest analytical grade commercially available and were purchased from Sigma.

2.2. Culture of Human Adipocytes and CM Generation. Subcutaneous adipose tissue was obtained from healthy lean or moderately overweight women $(n=23$, body mass index (BMI) $26.1 \pm 1.1$, and aged $36.6 \pm 2.0$ years) undergoing plastic surgery. The procedure was approved by the ethical committee of the Heinrich-Heine-University (Düsseldorf, Germany). All subjects were healthy and free of medication and had no evidence of metabolic diseases according to routine laboratory tests. Preadipocytes were isolated by collagenase digestion of adipose tissue as previously described by us [11]. Seeded preadipocytes were induced to differentiation into adipocytes over 15 days as previously described by us [10]. The degree of differentiation was determined by oil red staining and induction of adiponectin. Differentiated adipocytes were used for the generation of adipocyte-CM, as recently described by us [12]. Briefly, CM was generated by culturing adipocytes for $48 \mathrm{~h}$ in VSMC basal medium (PromoCell) with addition of $50 \mathrm{ng} / \mathrm{mL}$ amphotericin b and $50 \mu \mathrm{g} / \mathrm{mL}$ gentamycin. Each CM was tested for its proliferative effect and the content of adiponectin (negatively correlated to proliferation) [10]. A more-detailed characterization of CM was described previously by us $[12,13]$. CM generated from in vitro differentiated adipocytes was used for all experiments presented in Figures 1 and 2.

2.3. Generation of CM from Explants of SAT, VAT, and PAT. Paired biopsies from subcutaneous (SAT) und visceral adipose tissue (VAT) were obtained from lean patients without diabetes $(n=10$, body mass index (BMI) of $21 \pm 1 \mathrm{~kg} / \mathrm{m}^{2}$ aged $67 \pm 6$ ), obese patients without diabetes $\left(n=11\right.$, BMI of $38 \pm 3 \mathrm{~kg} / \mathrm{m}^{2}$ aged $56 \pm 6$ years $)$ and obese patients with type 2 diabetes $(n=6$, BMI of $37 \pm$ $6 \mathrm{~kg} / \mathrm{m}^{2}$ aged $56 \pm 5$ years) undergoing elective surgery such as hernia, gall bladder surgery, and other noninflammatory and nonmalignant causes. Human perivascular (PAT) and SAT biopsies were obtained from patients with type 2 diabetes ( $n=5$, BMI $29 \pm 7 \mathrm{~kg} / \mathrm{m}^{2}$, aged $68 \pm 9$ years) and patients without type 2 diabetes $\left(n=9\right.$, BMI $28 \pm 4 \mathrm{~kg} / \mathrm{m}^{2}$, aged $69 \pm 12$ years) undergoing coronary artery bypass surgery. Information on the diabetes status was obtained from the medical records of the patients. PAT was collected from the coronary artery and used to generate CM as described $[14,15]$ using $100 \mathrm{mg}$ of adipose tissue explant to generate $1 \mathrm{~mL}$ of CM. After $24 \mathrm{~h}, \mathrm{CM}$ was collected and stored in aliquots at $-80^{\circ} \mathrm{C}$ until further use. CM generated from SAT and VAT was used for all experiments presented in Figure 3, and CM generated from SAT and PAT was used for all experiments presented in Figure 4. 


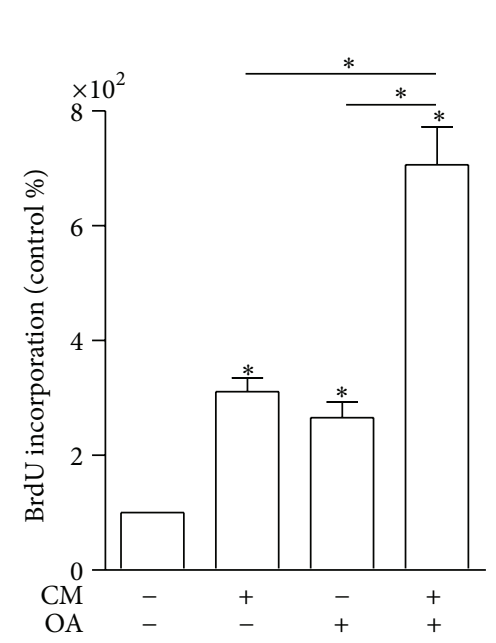

(a)

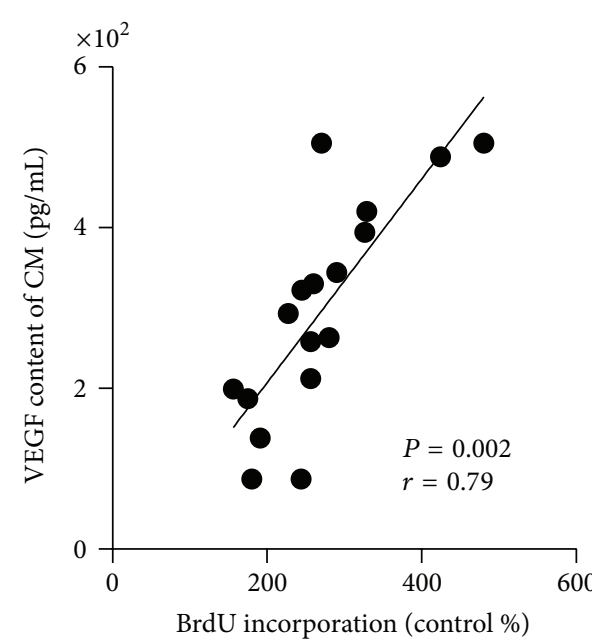

(b)

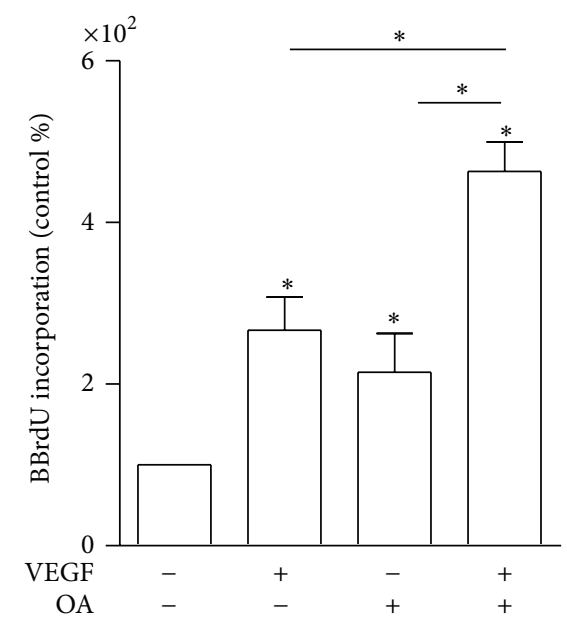

(c)

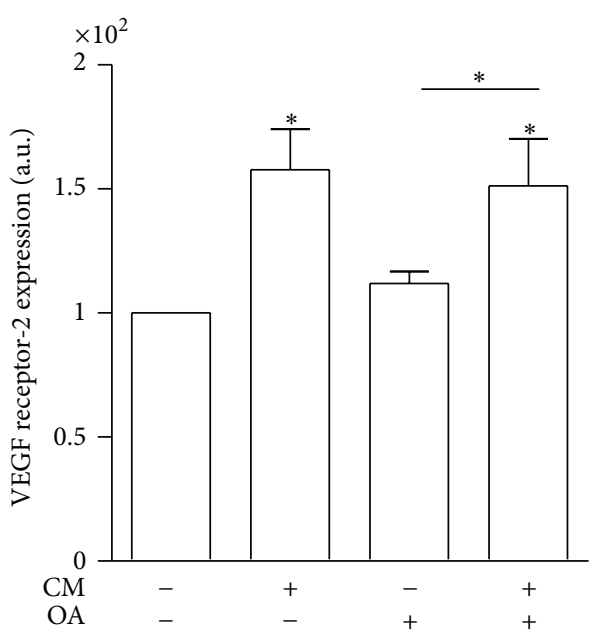

(f)

FIGURE 1: CM-induced proliferation is mediated by VEGF. (a) VSMC were serum starved for $24 \mathrm{~h}$ and subsequently incubated with CM, $100 \mu \mathrm{mol} / \mathrm{L} \mathrm{OA}$, or the combination of CM and OA in the presence of BrdU for $18 \mathrm{~h}$. Incorporation of BrdU into DNA was measured. Data are expressed relative to control, taken as $100 \%$. Data are mean values \pm SEM of three independent experiments. ${ }^{*} P<0.05$ compared to control or designated treatment. (b) The proliferative effect of CM correlates significantly with its VEGF content as measured by ELISA. Statistical result of linear regression analysis is indicated in the graph. (c) VSMC were serum starved for $24 \mathrm{~h}$ and subsequently incubated with $250 \mathrm{pg} / \mathrm{mL}$ VEGF, $100 \mu \mathrm{mol} / \mathrm{L} \mathrm{OA}$, or the combination of VEGF and OA in the presence of BrdU for $18 \mathrm{~h}$. Incorporation of BrdU into DNA was measured. Data are expressed relative to control, taken as $100 \%$. Data are mean values \pm SEM of three independent experiments. ${ }^{*} P<0.05$ compared to control or designated treatment. (d-f) VSMC were treated with CM, OA, and their combination for $24 \mathrm{~h}$. Total cell lysates were resolved by SDS-PAGE and immunoblotted with specific VEGF-R1 and -2 antibodies. A representative blot is shown. Data are mean values \pm SEM of three independent experiments. All data were normalized to the level of actin expression and are expressed relative to the control.

2.4. Culture of Human Vascular Smooth Muscle Cells (VSMC). Primary human coronary artery VSMC were obtained from PromoCell (Heidelberg, Germany). VSMC from four different donors (Caucasian, male, 23, 31, and 40 years old; female, 56 years old) were used as subconfluent cells of passage 3 . Cells were characterized as VSMC by morphologic criteria and by immunostaining with smooth muscle $\alpha$-actin.

2.5. In Vitro Analysis of VSMC Proliferation. To monitor DNA synthesis, VSMC were seeded in 96-well culture dishes and allowed to attach for $24 \mathrm{~h}$, followed by serum starvation for an additional $24 \mathrm{~h}$ period. Cells were then stimulated for $18 \mathrm{~h}$ as outlined above in the presence of BrdU $(10 \mu \mathrm{M})$. 10.000 VSMC per $15 \mathrm{~mm}^{2}$ well were incubated with the CM of 35.000 adipocytes. The BrdU ELISA Kit was used to determine proliferation according to the manufacturer's protocol. Signals were visualized and evaluated on a LUMI Imager work station (Boehringer, Mannheim, Germany).

2.6. Immunoblotting. VSMC were treated as indicated and lysed in a buffer containing $50 \mathrm{mM}$ HEPES, pH 7.4, 1\% 


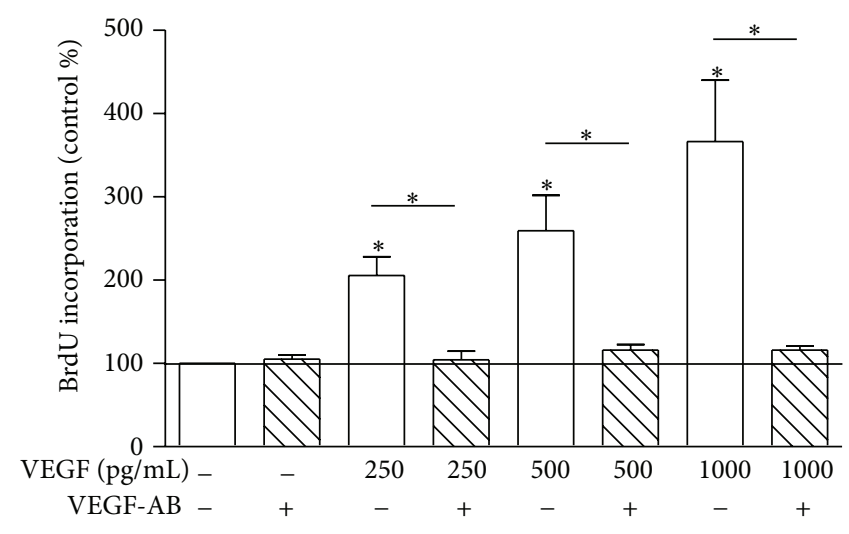

(a)

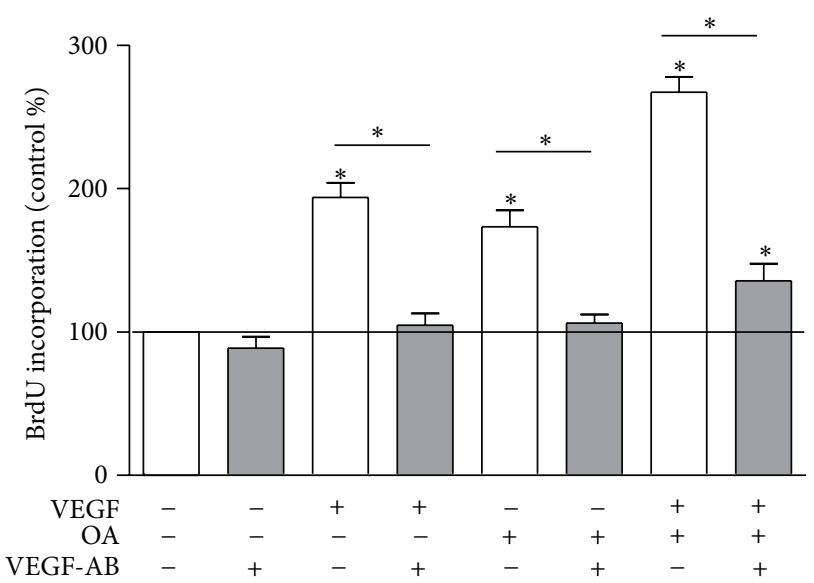

(c)

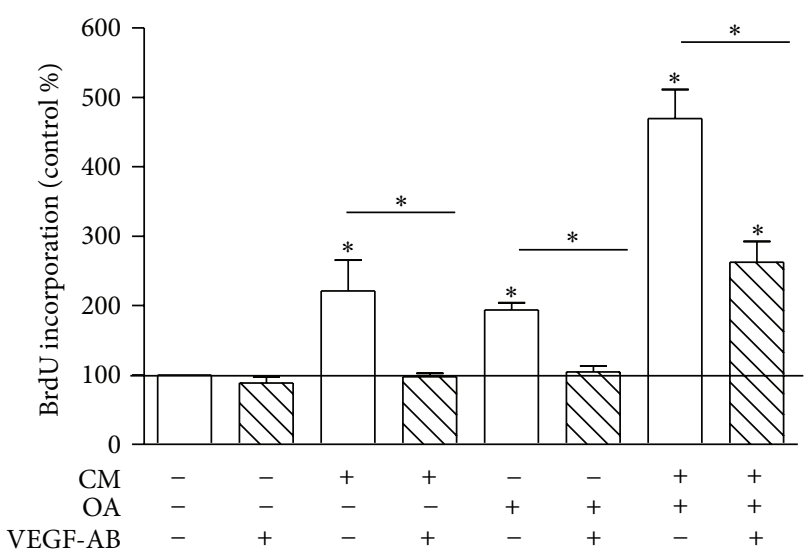

(b)
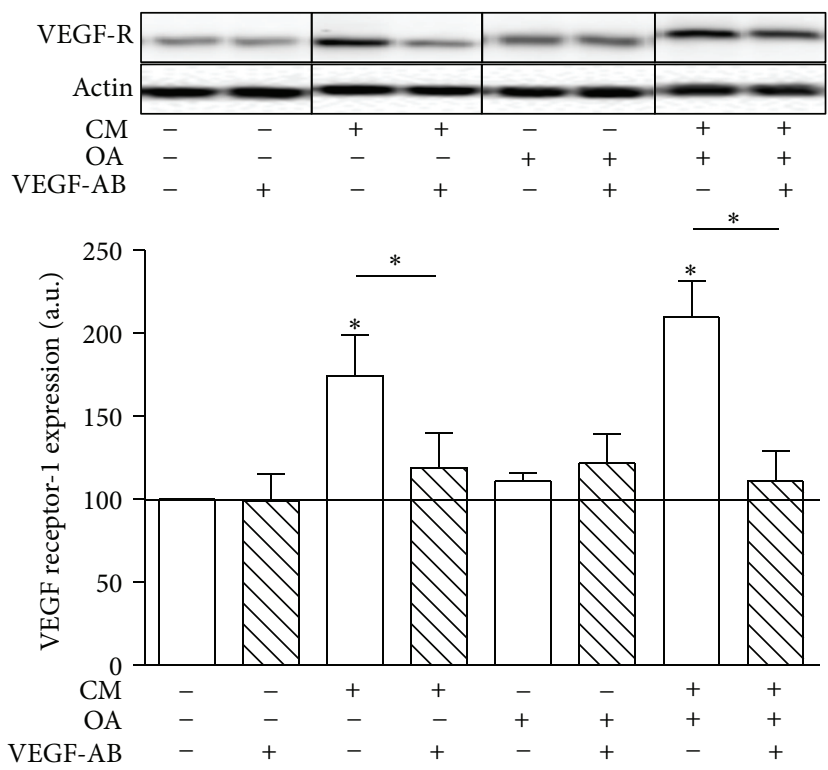

(d)

FIGURE 2: VEGF neutralization prevents CM- and OA-induced proliferation. Cells were treated with 250, 500, and 1000 pg/mL VEGF (a), $\mathrm{CM}, \mathrm{OA}$ and CMOA (b and d), and accordingly VEGF, OA, and VEGFOA (c) as described in the legend to Figure 1 in the presence or absence of a specific neutralizing VEGF antibody for $24 \mathrm{~h}$. (a-c) BrdU incorporation into DNA was determined as described in the legend to Figure 1. Data are expressed relative to control. (d) Total cell lysates were resolved by SDS-PAGE and immunoblotted with a specific VEGF-R1 antibody. For VEGF-R1, lanes were excised from a single western blot and displayed in the present order. Data are mean values \pm SEM of three independent experiments. All data were normalized to the level of actin expression and are expressed relative to the control. ${ }^{*} P<0.05$ compared to untreated control or designated data.

TritonX100, complete protease inhibitor, and PhosStop phosphatase inhibitor cocktail. After incubation for $2 \mathrm{~h}$ at $4^{\circ} \mathrm{C}$, the suspension was centrifuged at $10.000 \times \mathrm{g}$ for $15 \mathrm{~min}$. Thereafter, $5 \mu \mathrm{g}$ protein of lysates were separated by SDS-PAGE using 10\% horizontal gels and transferred to polyvinylidene fluorid filters in a semidry blotting apparatus [16]. Filters were blocked with Tris-buffered saline, containing $0.1 \%$ Tween and $5 \%$ nonfat dry milk, and subsequently incubated overnight with a 1:1000 dilution of the appropriate antibodies. After washing, filters were incubated with secondary HRP-coupled antibody and processed for enhanced chemiluminescence detection using Immobilon HRP substrate (Millipore, Billerica, USA). Signals were visualized and evaluated on a VersaDoc work station (Bio-Rad Laboratories, Munich, Germany).

2.7. Presentation of Data and Statistics. Data are expressed as mean \pm SEM. One-way ANOVA (post hoc test: Bonferroni's multiple comparison test) was used to determine statistical significance. All statistical analyses were done using Prism (GraphPad, La Jolla, USA) considering a $P$ value of less than 


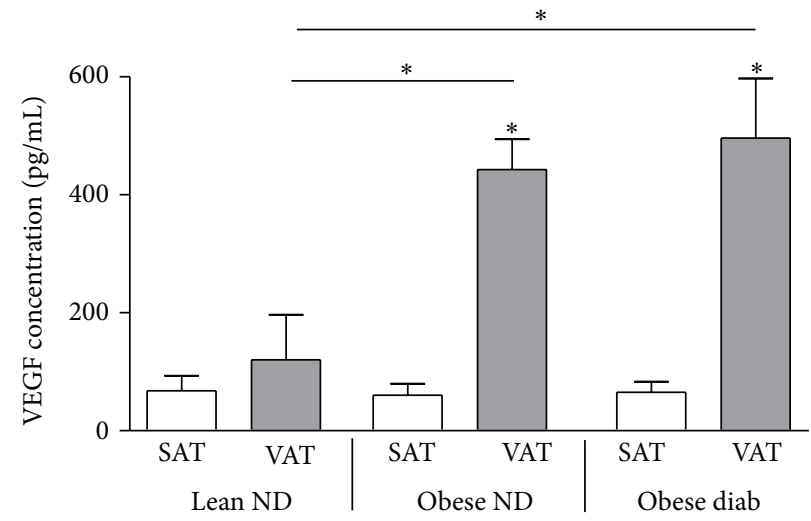

(a)

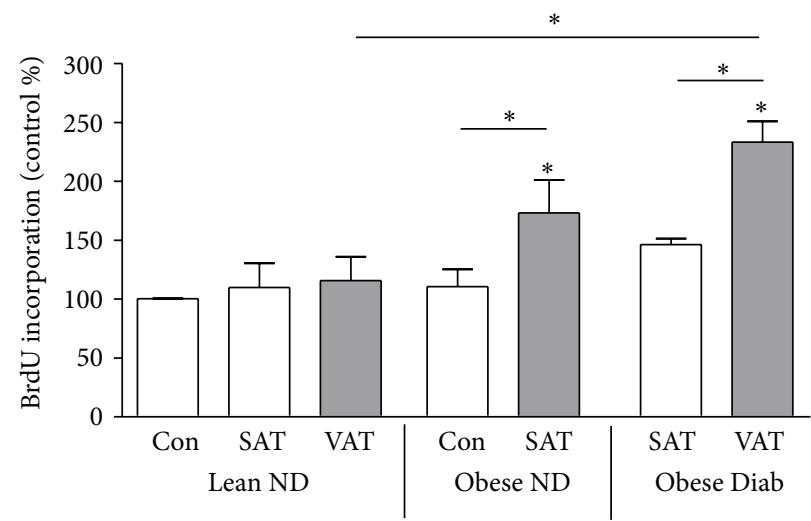

(b)

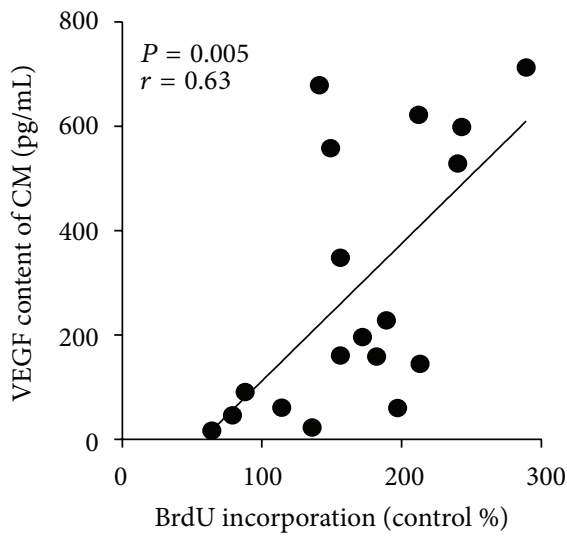

(c)

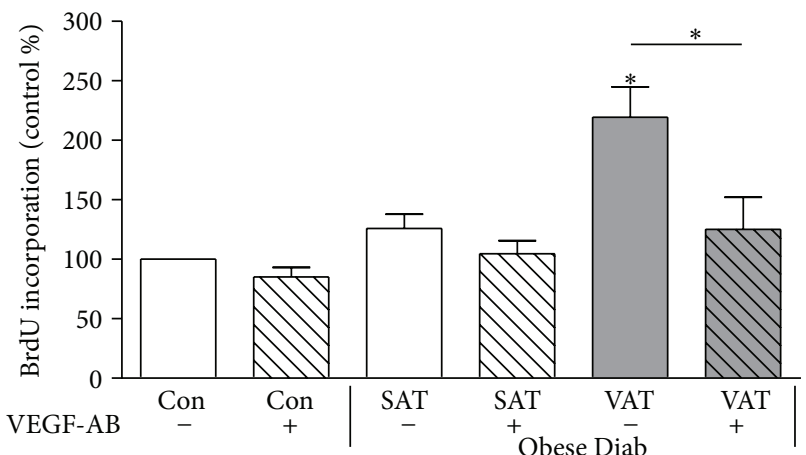

(d)

FIGURE 3: VEGF release is higher from VAT of obese subjects correlating with induction of VSMC proliferation. (a) VEGF content of CM from VAT and SAT was measured in duplicates by ELISA. (b and d) VSMC were treated with CM from paired SAT and VAT of lean nondiabetic (ND), obese ND, and obese type 2 diabetes (Diab) patients for $24 \mathrm{~h}$. For (d), VSMC were treated in the presence or absence of a VEGFneutralizing antibody. VSMC proliferation was determined by measuring the incorporation of BrdU into DNA. Data are expressed relative to the basal control value, which was set as $100 \%$. Data are presented as mean \pm SEM from five independent experiments. For (d), all data were normalized to the level of actin expression and are expressed relative to the control ${ }^{*} P<0.05$ compared to control or designated data. For (d), lanes were excised from a single western blot and displayed in the presented order. (c) The proliferative effect of CM from VAT correlates significantly with its VEGF content as measured by ELISA. Statistical result of linear regression analysis is indicated in the graph.

0.05 as statistically significant. Corresponding significance levels are indicated in the figures.

\section{Results}

3.1. VEGF Content of CM Correlates with CM-Induced Proliferation. We have previously shown that CM from in vitro differentiated human adipocytes induces proliferation of human coronary artery VSMC with a large majority of the CMs tested inducing a prominent proliferation of more than 2 -fold compared to control [10]. While CM and OA induce similarly strong proliferation of VSMC, their combination increased the proliferation in a synergistic way (Figure 1(a)), as reported previously [10]. The proliferative effect of CM negatively correlated with its adiponectin concentrations, but no correlation was found with IL-6 in CM [10]. Correlating $\mathrm{CM}$ content of growth inducing factors to $\mathrm{CM}$-induced proliferation, we found that CM-induced proliferation strongly correlated with the VEGF content of the respective CM (Figure 1(b)). When replacing CM by VEGF at the average concentration found in the tested CM $(250 \mathrm{pg} / \mathrm{mL}$ human recombinant VEGF), the proliferative effect of CM could be mimicked (Figure 1(c)) in accordance with previous data [10]. Treatment with CM, OA, or the combination of CMOA induced VEGF secretion of VSMC (control $178 \pm 20 \mathrm{pg} / \mathrm{mL}$, CM-treated $507 \pm 20 \mathrm{pg} / \mathrm{mL}$, OA-treated $414 \pm 52 \mathrm{pg} / \mathrm{mL}$, and CMOA-treated cells $1109 \pm 69 \mathrm{pg} / \mathrm{mL}, n=4)$ as previously described [10]. These data suggest that VSMC significantly contribute to proliferation by releasing VEGF for autocrine/paracrine stimulation. CM and the combination of CMOA also increased the expression of VEGF-R1 and -2, whereas OA had no effect on its expression (Figures 1(d)$1(f))$.

3.2. VEGF Is an Important Factor for CM- and OA-Induced VSMC Proliferation. In order to assess the importance of 


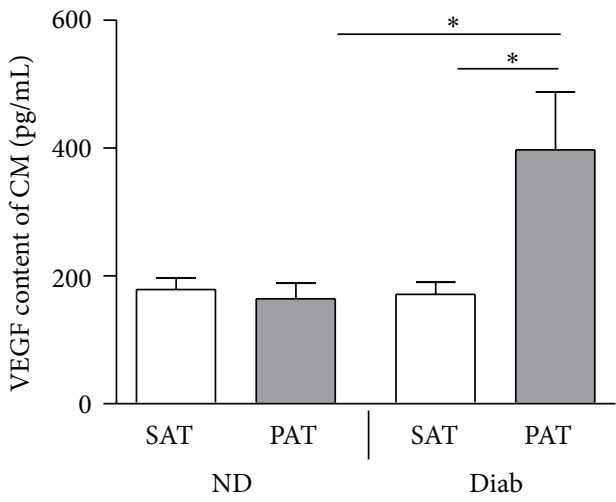

(a)

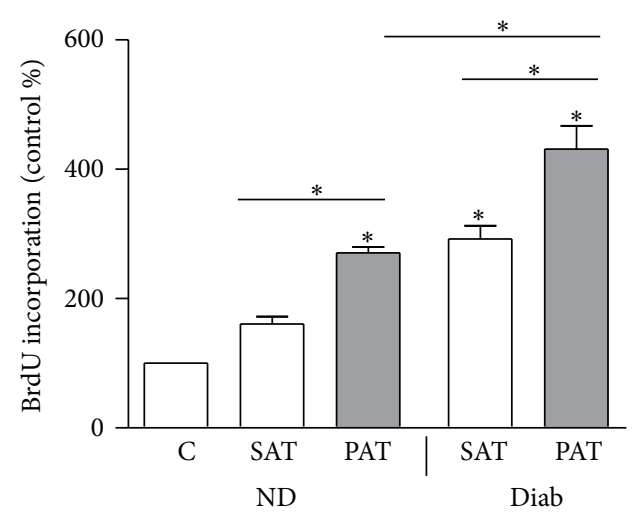

(b)

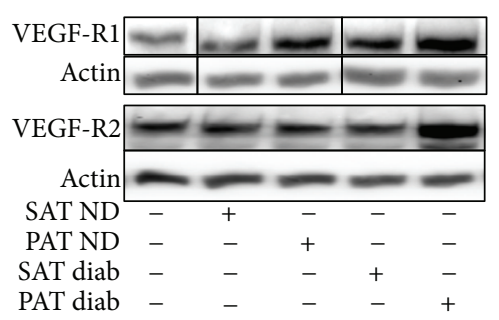

(c)

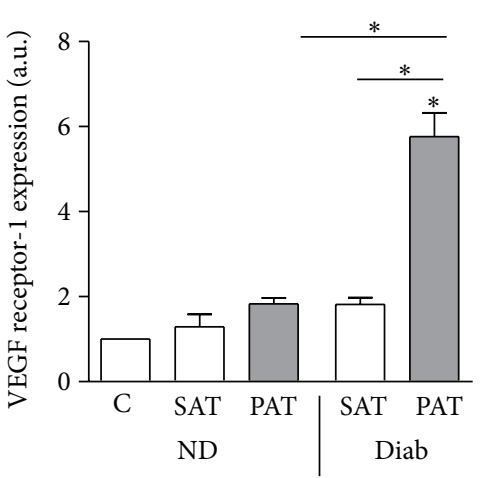

(d)

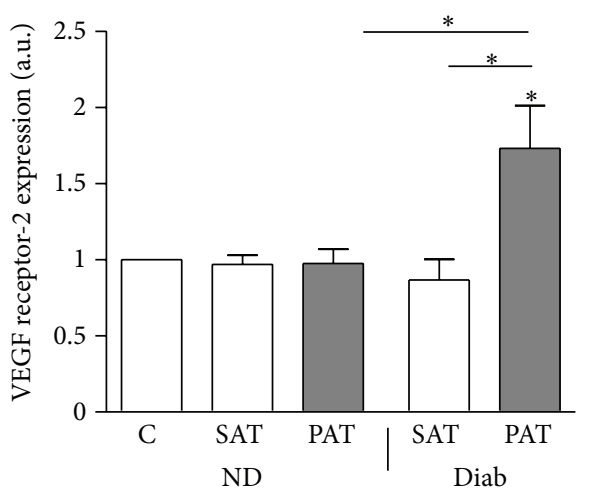

(e)

FIGURE 4: VEGF release is higher from PAT of type 2 diabetic patients correlating with VSMC proliferation and VEGF-R1/2 expression. (a) VEGF content of CM from PAT and SAT was measured in duplicates by ELISA. (b) VSMC were treated with CM from paired SAT and PAT of patients with type 2 diabetes (Diab) and nondiabetics controls (ND) for $24 \mathrm{~h}$. The proliferation was determined by measuring the incorporation of BrdU into DNA. Data are expressed relative to the basal control value, which was set as $100 \%$. Data are presented as mean \pm SEM from five independent experiments. (c-e) Total cell lysates were resolved by SDS-PAGE and immunoblotted with specific VEGF-R1 and 2 antibodies. Data are mean values \pm SEM of three to five independent experiments. All data were normalized to the level of actin expression and are expressed relative to the control. ${ }^{*} P<0.05$ compared to control or designated data.

VEGF in CM-induced proliferation, VEGF was neutralized with a specific antibody. To establish effective VEGF neutralization, VEGF was applied at concentrations ranging from $250 \mathrm{pg} / \mathrm{mL}$ to $1 \mu \mathrm{g} / \mathrm{mL}$, which mimics both the average VEGF concentration in $\mathrm{CM}$ and spans to the highest VEGF release by VSMC observed after CMOA treatment (Figure 2(a)). Neutralizing VEGF prevented the CM-, VEGF-, and OAinduced proliferation completely (Figures 2(b) and 2(c)), underlining our hypothesis that CM and OA increase VSMC proliferation via VEGF. VEGF blocking significantly reduced the proliferative effect of the combination CMOA, which is still elevated compared to untreated control (Figure 2(b)), illustrating that under this condition VEGF is not the only factor involved in the proliferative effect. Furthermore, the induction of VEGF-R1 by CM and the combination of CM and OA was also completely prevented by VEGF blocking (Figure 2(d)). pared to SAT and VAT from Lean Controls Correlating with
VSMC Proliferation. VEGF release from VAT was significantly higher compared to SAT in both obese subjects without type 2 diabetes and with type 2 diabetes (Figure 3(a)). On the other hand, VEGF release was low and comparable from VAT and SAT of lean subjects. Accordingly, VSMC proliferation was induced by CM from VAT of obese subjects, but not by CM from SAT, VAT from lean patients, and SAT from obese patients (Figure 3(b)). Similar to CM derived from human adipocytes (see Figure 1(b)), the VEGF content of CM from VAT correlated significantly with the proliferative effect of the respective CM (Figure 3(c)). However, VEGF content of CM from SAT was not correlated to proliferation (data not shown). Neutralization of VEGF prevented proliferation induced by CM from VAT of obese subjects with type 2 diabetes (Figure 3(d)).

3.4. PAT of Type 2 Diabetic Patients Releases Increased Amounts of VEGF and Induces Significant Proliferation of VSMC. The release of VEGF was comparable from SAT and PAT of the nondiabetic subjects and from SAT of patients 
with type 2 diabetes (Figure 4(a)) while its release by PAT of type 2 diabetic patients was significantly increased. Accordingly, CM from PAT of type 2 diabetic patients induced the strongest proliferative effect on VSMC (Figure 4(b)). In both groups of patients, CM from PAT induced a stronger proliferation as compared to CM from SAT. Furthermore, CM from PAT of patients with type 2 diabetes induced a 2to 4 -fold increase of VEGF-R1 and -2 expression in VSMC (Figures 4(c) and 4(d)). In contrast, PAT of nondiabetics exerted a comparable effect to the respective SAT. Our findings suggest that VEGF may be an important adipokine produced in PAT especially from patients with type 2 diabetes that might directly induce proliferation and expression of VEGF-R1 in VSMC.

\section{Discussion}

Obesity is strongly related to the development of cardiovascular diseases, and adipokines have been suggested to be a molecular link in this relationship [4, 17]. This study was designed to elucidate mechanisms on how the secretory output from adipose tissue is related to VSMC proliferation. We could show in a previous study that CM of in vitro differentiated adipocytes induces proliferation of VSMC in negative correlation to the adiponectin content of CM [10]. Searching for an active component of CM being responsible for VSMC proliferation, we found VEGF in CM to be significantly correlated with proliferation. VEGF is traditionally known as an endothelial cell-specific growth factor, which modulates vascular disease by inducing endothelial proliferation mainly through the VEGF-R2 [18]. However, an increase of VEGF and VEGF-Rs could be observed in other injured cells of the arterial wall like monocytes and VSMC [19]. Vascular inflammation and the proliferation of endothelial cells as well as VSMC are enhanced through angiotensin II-induced VEGF release and expression of VEGF-R [20-23].

Here, we report that $\mathrm{CM}$ and $\mathrm{OA}$ as well as their combination induce release of VEGF also by VSMC, which in turn might autostimulate proliferation. This effect is similar to hypoxia-induced proliferation of VSMC, where an autocrine proliferative action of VEGF has been described [24]. In addition, it has been described that proinflammatory stimulation with angiotensin II and IL-1beta and activation of JNK induce VEGF release by VSMC $[25,26]$. It might be speculated that proinflammatory adipokines present in $\mathrm{CM}$ are, therefore, responsible for the induction of VEGF. Furthermore, VEGF might autostimulate its release which might be worth to study in the future. In addition to stimulating the release of VEGF from VSMC, CM induced VEGF-R1 and -2 expression which mediate proliferation and migration of VSMC [27]. Our data suggest that both VEGF-Rs are regulated by VEGF itself, as neutralizing VEGF prevented CM-induced expression of these receptors in parallel to proliferation. In fact, a regulation of VEGF-R2 by VEGF has already been proposed [28]. Interestingly, OA stimulates VEGF secretion but not the expression of VEGF-Rs. As oleic acid has complex effects on VSMC, it can only be speculated that it directly or indirectly affects VEGF-R expression by still unknown mechanisms.
VEGF is a proinflammatory factor $[29,30]$, and we previously demonstrated that $\mathrm{CM}$ activates NF- $\kappa \mathrm{B}$ signaling that is essential for CM-induced proliferation [10]. VEGF is not primarily activating NF- $\kappa \mathrm{B}$ at concentrations measured and used in this study [29]. However, CM contains various adipokines including MCP-1 and chemerin $[12,31]$ that have been described to strongly activate NF- $\kappa$ B. Activated NF$\kappa \mathrm{B}$ could then in turn lead to increased VEGF expression and release as described in the literature [32]. Thereby, proinflammatory factors in $\mathrm{CM}$ may contribute to proliferation by inducing VEGF via NF- $\kappa$ B. Blocking VEGF with a specific neutralizing VEGF-antibody reduced the CM, OA, and VEGF-induced proliferation of VSMC completely. In contrast, the strong proliferative effect of the combination of $\mathrm{CM}$ and $\mathrm{OA}$ was not totally prevented, illustrating that VEGF is not the only important factor for the synergism of CMOA. As CM contains various adipokines such as IL-6, IL-8, or MCP-1 [12], it is possible that some of these factors induce proliferation in addition to VEGF and that these factors might also be involved in the synergistic effects of $\mathrm{CM}$ and OA. Several adipokines such as leptin and resistin affected VSMC proliferation and function $[33,34]$ but are not probable to play a role in this scenario. Leptin release by adipocytes differentiated in vitro is too low to explain CMinduced proliferation [35], and resistin is only secreted from mouse but not from human adipocytes [12].

We show here that VEGF release is higher from VAT of obese subjects irrespective of their metabolic status. Previous studies have shown that VEGF expression is already increased along with increased inflammatory adipokines in VAT of children compared to SAT [36]. In adults, VEGF expression in obese patients is higher in VAT as compared to SAT [37]. Increased VEGF expression might be associated with higher inflammation in VAT as VEGF expression, and secretion is stimulated by inflammatory cytokines such as IL-6 [38]. Recently, it has also been suggested that hypoxia can induce VEGF expression in expanded adipose tissue [39]. VEGF in adipose tissue is recently discussed both as a "bad" and a "good" guy. One important function of VEGF within adipose tissue is to stimulate angiogenesis, which is crucial for increasing blood capillaries in expanding adipose tissue by stimulating endothelial cell growth. Here, VEGF is described as beneficial for preventing adipose tissue hypoxia and thus inflammation. In fact, several groups have demonstrated that inducible overexpression of VEGF in adipose tissue prevents obesity-induced hypoxia and inflammation in adipose tissue in parallel to improved metabolic phenotype [40-42]. Conversely, adipose-specific ablation of VEGF decreases vascularization and increases inflammation together with deteriorated metabolic control in high-fat diettreated mice [42]. In the context of existing obesity, increased VEGF expression is rather seen as another stimulator of proinflammatory adipocytes further deteriorating adipose tissue function. Here, inducible ablation of VEGF in adipose tissue protects from obesity and induces brown adipose tissue within white adipose tissue in mice [43]. Taken together with another similar study using different mouse models [41], it has been suggested that the effects of VEGF within adipose are context dependent. While high VEGF expression 
in already expanded adipose tissue deteriorates metabolic control, increased angiogenic activity by VEGF induction during expansion of adipose tissue is beneficial to maintain normal metabolic function. As no detailed studies on VEGF expression of human adipose tissue during obesity development exist, further work is needed to expand this view to the human situation.

It has been proposed that PAT is involved in a paracrine crosstalk with cells of the vascular wall and that adipokines are major players in mechanisms linking PAT to inflammation and vascular dysfunction $[44,45]$. Here, we demonstrate that PAT from patients with type 2 diabetes is characterized by a significantly higher VEGF release as compared to SAT and also compared to PAT from nondiabetics. Higher VEGF release is paralleled by a stronger induction of VEGF-R1 and -2. The use of paired biopsies from human SAT and PAT further strengthens the notion of VEGF release from PAT for a potential paracrine crosstalk. The VEGF content of CM from SAT and PAT from patients without diabetes as well as SAT from type 2 diabetic subjects was similar but did not have the same effect on VSMC proliferation. It should be noted that the use of adipose tissue explants is a more complex setting than using CM from adipocytes as the secretory output from adipose tissue explants also contains factors derived from other cell types present in adipose tissue as preadipocytes and macrophages. Further work will be needed to elucidate the differences in adipokine expression and secretion between PAT and other fat depots in health and disease in order to identify other putative factors responsible for PAT-induced vascular dysfunction.

A limitation of the study is that the small amounts of CM that could be prepared from the surgical biopsies from PAT disallowed experiments assessing the impact of VEGF neutralizing antibodies. In this respect, it should be noted that the secretory profile of human adipocytes and adipose tissue biopsies is complex, as illustrated for example, by disease and depot-specific differences in the adipokines released $[15,46]$. The fact that VEGF content and the potential of PAT-derived conditioned media to induce VSMC proliferation do not seem to fully correlate suggests that a contribution of other adipokines cannot be excluded. For example, activin A, which is released in substantial amounts from epicardial adipose tissue, which is highly related to PAT $[15,47]$, has also been linked to VSMC proliferation [48]. Notably, activin A release is elevated in epicardial adipose tissue from patients with type 2 diabetes and may thus explain higher proliferation of VSMC treated with CM from PAT of patients with type 2 diabetes and PAT of patients without diabetes compared to SAT of patients without diabetes despite similar VEGF content in these CMs. Differently from PAT-induced VSMC proliferation, VAT-induced proliferation could be explained by higher VEGF content in CM from VAT of obese patients with type 2 diabetes and completely prevented by VEGF neutralization. Therefore, further studies remain required to further detail the impact of PAT-derived adipokines on VSMC proliferation.

In conclusion, our results demonstrate that VEGF is mediating CM-induced proliferation of VSMC. As an adipokine and growth factor that is released in high amounts from VAT of obese patients and PAT of patients with type 2 diabetes compared to SAT of lean and nondiabetic controls, VEGF might be a link between adipose tissue inflammation and abnormal VSMC proliferation.

\section{Conflict of Interests}

The authors have no conflict of interests to declare.

\section{Acknowledgments}

This work was supported by the Bundesministerium für Gesundheit, the Commission of the European Communities (Collaborative Project ADAPT, Contract No. HEALTHF2-2008-201100), and the German Research Council (SE1922/2-1). The authors wish to thank the Department of Plastic Surgery, Florence-Nightingale-Hospital Düsseldorf, for support in obtaining adipose tissue samples for adipocyte isolation. The technical assistance of Andrea Cramer and Angelika Horrighs and the secretarial assistance of Birgit Hurow are gratefully acknowledged.

\section{References}

[1] N. Ouchi, J. L. Parker, J. J. Lugus, and K. Walsh, "Adipokines in inflammation and metabolic disease," Nature Reviews Immunology, vol. 11, no. 2, pp. 85-97, 2011.

[2] S. Lehr, S. Hartwig, and H. Sell, "Adipokines: a treasure trove for the discovery of biomarkers for metabolic disorders," Proteomics, vol. 6, no. 1-2, pp. 91-101, 2012.

[3] H. Sell and J. Eckel, "Adipose tissue inflammation: novel insight into the role of macrophages and lymphocytes," Current Opinion in Clinical Nutrition and Metabolic Care, vol. 13, no. 4, pp. 366-370, 2010.

[4] A. Taube, R. Schlich, H. Sell, K. Eckardt, and J. Eckel, "Inflammation and metabolic dysfunction: links to cardiovascular diseases," American Journal of Physiology, vol. 302, pp. H2148H2165, 2012.

[5] H. Ruan and H. F. Lodish, "Insulin resistance in adipose tissue: direct and indirect effects of tumor necrosis factor- $\alpha$," Cytokine and Growth Factor Reviews, vol. 14, no. 5, pp. 447-455, 2003.

[6] F. B. Hu, W. C. Willett, T. Li, M. J. Stampfer, G. A. Colditz, and J. E. Manson, "Adiposity as compared with physical activity in predicting mortality among women," The New England Journal of Medicine, vol. 351, no. 26, pp. 2694-2703, 2004.

[7] R. M. van Dam, W. C. Willett, J. E. Manson, and F. B. Hu, “The relationship between overweight in adolescence and premature death in women," Annals of Internal Medicine, vol. 145, no. 2, pp. 91-97, 2006.

[8] P. Mathieu, P. Poirier, P. Pibarot, I. Lemieux, and J.-P. Després, "Visceral obesity the link among inflammation, hypertension, and cardiovascular disease," Hypertension, vol. 53, no. 4, pp. 577-584, 2009.

[9] G. Iacobellis and A. M. Sharma, "Epicardial adipose tissue as new cardio-metabolic risk marker and potential therapeutic target in the metabolic syndrome," Current Pharmaceutical Design, vol. 13, no. 21, pp. 2180-2184, 2007.

[10] D. Lamers, R. Schlich, S. Greulich, S. Sasson, H. Sell, and J. Eckel, "Oleic acid and adipokines synergize in inducing proliferation and inflammatory signalling in human vascular smooth 
muscle cells," Journal of Cellular and Molecular Medicine, vol. 15, no. 5, pp. 1177-1188, 2011.

[11] H. Hauner, T. Petruschke, M. Russ, K. Rohrig, and J. Eckel, "Effects of tumour necrosis factor alpha $(\mathrm{TNF} \alpha)$ on glucose transport and lipid metabolism of newly differentiated human fat cells in cell culture," Diabetologia, vol. 38, no. 7, pp. 764-771, 1995.

[12] D. Dietze-Schroeder, H. Sell, M. Uhlig, M. Koenen, and J. Eckel, "Autocrine action of adiponectin on human fat cells prevents the release of insulin resistance-inducing factors," Diabetes, vol. 54, no. 7, pp. 2003-2011, 2005.

[13] H. Sell, K. Eckardt, A. Taube et al., "Skeletal muscle insulin resistance induced by adipocyte-conditioned medium: underlying mechanisms and reversibility," American Journal of Physiology, vol. 294, no. 6, pp. E1070-E1077, 2008.

[14] S. Greulich, D. H. de Wiza, S. Preilowski et al., "Secretory products of guinea pig epicardial fat induce insulin resistance and impair primary adult rat cardiomyocyte function," Journal of Cellular and Molecular Medicine, vol. 15, no. 11, pp. 2399-2410, 2010.

[15] S. Greulich, B. Maxhera, G. Vandenplas et al., "Secretory products from epicardial adipose tissue of patients with type 2 diabetes mellitus induce cardiomyocyte dysfunction," Circulation, vol. 126, pp. 2324-2334, 2012.

[16] A. Wichelhaus, M. Russ, S. Petersen, and J. Eckel, "G protein expression and adenylate cyclase regulation in ventricular cardiomyocytes from STZ-diabetic rats," American Journal of Physiology, vol. 267, no. 2, pp. H548-H555, 1994.

[17] P. Mathieu, I. Lemieux, and J.-P. Després, "Obesity, inflammation, and cardiovascular risk," Clinical Pharmacology and Therapeutics, vol. 87, no. 4, pp. 407-416, 2010.

[18] M. Shibuya, "Differential roles of vascular endothelial growth factor receptor-1 and receptor-2 in angiogenesis," Journal of Biochemistry and Molecular Biology, vol. 39, no. 5, pp. 469-478, 2006.

[19] Q. Zhao, K. Egashira, K.-I. Hiasa et al., "Essential role of vascular endothelial growth factor and Flt-1 signals in neointimal formation after periadventitial injury," Arteriosclerosis, Thrombosis, and Vascular Biology, vol. 24, no. 12, pp. 2284-2289, 2004.

[20] Q. Zhao, M. Ishibashi, K.-I. Hiasa, C. Tan, A. Takeshita, and K. Egashira, "Essential role of vascular endothelial growth factor in angiotensin II-induced vascular inflammation and remodeling," Hypertension, vol. 44, no. 3, pp. 264-270, 2004.

[21] H. Kitayama, Y. Maeshima, Y. Takazawa et al., "Regulation of angiogenic factors in angiotensin II infusion model in association with tubulointerstitial injuries," American Journal of Hypertension, vol. 19, no. 7, pp. 718-727, 2006.

[22] D. Herr, M. Rodewald, H. M. Fraser et al., "Regulation of endothelial proliferation by the renin-angiotensin system in human umbilical vein endothelial cells," Reproduction, vol. 136, no. 1, pp. 125-130, 2008.

[23] P. Pan, H. Fu, L. Zhang et al., "Angiotensin II upregulates the expression of placental growth factor in human vascular endothelial cells and smooth muscle cells," BMC Cell Biology, vol. 11, article 36, 2010.

[24] M. Osada-Oka, T. Ikeda, S. Akiba, and T. Sato, "Hypoxia stimulates the autocrine regulation of migration of vascular smooth muscle cells via HIF-1 $\alpha$-dependent expression of thrombospondin-1," Journal of Cellular Biochemistry, vol. 104, no. 5, pp. 1918-1926, 2008.
[25] Y. D. Jung, W. Liu, N. Reinmuth et al., "Vascular endothelial growth factor is upregulated by interleukin- $1 \beta$ in human vascular smooth muscle cells via the P38 mitogen-activated protein kinase pathway," Angiogenesis, vol. 4, no. 2, pp. 155-162, 2001.

[26] S.-I. Miura, Y. Matsuo, and K. Saku, "Jun N-terminal kinase inhibitor blocks angiogenesis by blocking VEGF secretion and an MMP pathway," Journal of Atherosclerosis and Thrombosis, vol. 15, no. 2, pp. 69-74, 2008.

[27] S. Banerjee, S. Mehta, I. Haque et al., "VEGF-A165 induces human aortic smooth muscle cell migration by activating neuropilin-1-VEGFR1-PI3K axis," Biochemistry, vol. 47, no. 11, pp. 3345-3351, 2008.

[28] X.-H. Yang, X.-Y. Man, S.-Q. Cai, Y.-G. Yao, Z.-Y. Bu, and M. Zheng, "Expression of VEGFR-2 on HaCaT cells is regulated by VEGF and plays an active role in mediating VEGF induced effects," Biochemical and Biophysical Research Communications, vol. 349, no. 1, pp. 31-38, 2006.

[29] T. Marumo, V. B. Schini-Kerth, and R. Busse, "Vascular endothelial growth factor activates nuclear factor- $\kappa \mathrm{B}$ and induces monocyte chemoattractant protein-1 in bovine retinal endothelial cells," Diabetes, vol. 48, no. 5, pp. 1131-1137, 1999.

[30] I. Kim, S.-O. Moon, S. H. Kim, H. J. Kim, Y. S. Koh, and G. Y. Koh, "Vascular endothelial growth factor expression of intercellular adhesion molecule 1 (ICAM-1), vascular cell adhesion molecule 1 (VCAM-1), and E-selectin through nuclear factor- $\kappa \mathrm{B}$ activation in endothelial cells," Journal of Biological Chemistry, vol. 276, no. 10, pp. 7614-7620, 2001.

[31] H. Sell, J. Laurencikiene, A. Taube et al., "Chemerin is a novel adipocyte-derived factor inducing insulin resistance in primary human skeletal muscle cells," Diabetes, vol. 58, no. 12, pp. 27312740, 2009.

[32] S. Wang, Z. Liu, L. Wang, and X. Zhang, "NF-kappaB signaling pathway, inflammation and colorectal cancer," Cellular \& Molecular Immunology, vol. 6, pp. 327-334, 2009.

[33] P. Calabro, I. Samudio, J. T. Willerson, and E. T. H. Yeh, "Resistin promotes smooth muscle cell proliferation through activation of extracellular signal-regulated kinase $1 / 2$ and phosphatidylinositol 3-kinase pathways," Circulation, vol. 110, no. 21, pp. 33353340, 2004.

[34] L. Li, J.-C. Mamputu, N. Wiernsperger, and G. Renier, "Signaling pathways involved in human vascular smooth muscle cell proliferation and matrix metalloproteinase- 2 expression induced by leptin: inhibitory effect of metformin," Diabetes, vol. 54, no. 7, pp. 2227-2234, 2005.

[35] S. Famulla, D. Lamers, S. Hartwig et al., "Pigment epitheliumderived factor (PEDF) is one of the most abundant proteins secreted by human adipocytes and induces insulin resistance and inflammatory signaling in muscle and fat cells," International Journal of Obesity, vol. 35, no. 6, pp. 762-772, 2011.

[36] C. S. Tam, L. K. Heilbronn, C. Henegar et al., "An early inflammatory gene profile in visceral adipose tissue in children," International Journal of Pediatric Obesity, vol. 6, no. 2, pp. e360e363, 2011.

[37] S. Ledoux, I. Queguiner, S. Msika et al., "Angiogenesis associated with visceral and subcutaneous adipose tissue in severe human obesity," Diabetes, vol. 57, no. 12, pp. 3247-3257, 2008.

[38] G. Rega, C. Kaun, S. Demyanets et al., "Vascular endothelial growth factor is induced by the inflammatory cytokines interleukin- 6 and oncostatin $\mathrm{m}$ in human adipose tissue in vitro and in murine adipose tissue in vivo," Arteriosclerosis, Thrombosis, and Vascular Biology, vol. 27, no. 7, pp. 1587-1595, 2007. 
[39] A. M. Fusaru, C. G. Pisoschi, A. Bold et al., "Hypoxia induced VEGF synthesis in visceral adipose depots of obese diabetic patients," Romanian Journal of Morphology and Embryology, vol. 53, pp. 903-909, 2013.

[40] I. Elias, S. Franckhauser, T. Ferre et al., "Adipose tissue overexpression of vascular endothelial growth factor protects against diet-induced obesity and insulin resistance," Diabetes, vol. 61, pp. 1801-1813, 2012.

[41] K. Sun, I. W. Asterholm, C. M. Kusminski et al., "Dichotomous effects of VEGF-A on adipose tissue dysfunction," Proceedings of the National Academy of Sciences of the United States of America, vol. 109, no. 15, pp. 5874-5879, 2012.

[42] H. K. Sung, K. O. Doh, J. E. Son et al., "Adipose vascular endothelial growth factor regulates metabolic homeostasis through angiogenesis," Cell Metabolism, vol. 17, pp. 61-72, 2013.

[43] X. Lu, Y. Ji, L. Zhang et al., "Resistance to obesity by repression of VEGF gene expression through induction of brown-like adipocyte differentiation," Endocrinology, vol. 153, pp. 31233132, 2012.

[44] D. M. Ouwens, H. Sell, S. Greulich, and J. Eckel, "The role of epicardial and perivascular adipose tissue in the pathophysiology of cardiovascular disease," Journal of Cellular and Molecular Medicine, vol. 14, no. 9, pp. 2223-2234, 2010.

[45] R. I. Meijer, E. H. Serne, Y. M. Smulders, V. W. M. Van Hinsbergh, J. S. Yudkin, and E. C. Eringa, "Perivascular adipose tissue and its role in type 2 diabetes and cardiovascular disease," Current Diabetes Reports, vol. 11, no. 3, pp. 211-217, 2011.

[46] S. Lehr, S. Hartwig, D. Lamers et al., "Identification and validation of novel adipokines released from primary human adipocytes," Molecular \& Cellular Proteomics, vol. 11, no. 1, Article ID M111.010504, 2012.

[47] N. Venteclef, V. Guglielmi, E. Balse et al., "Human epicardial adipose tissue induces fibrosis of the atrial myocardiumthrough the secretion of adipo-fibrokines," European Heart Journal. In press.

[48] S. H. Cho, Z. Yao, S.-W. Wang et al., "Regulation of activin A expression in mast cells and asthma: its effect on the proliferation of human airway smooth muscle cells," Journal of Immunology, vol. 170, no. 8, pp. 4045-4052, 2003. 


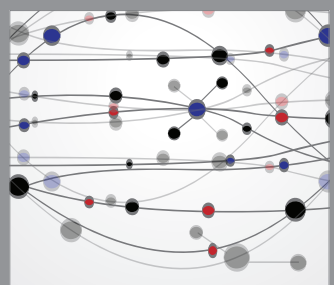

The Scientific World Journal
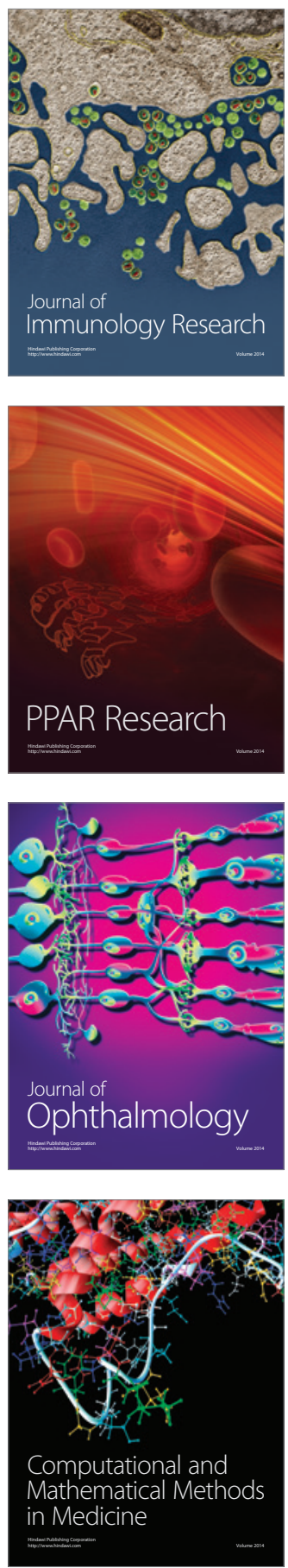

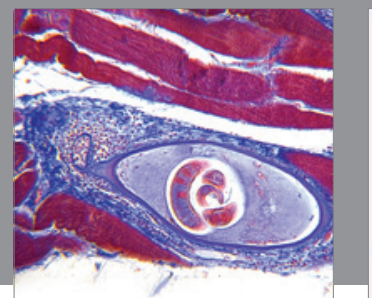

Gastroenterology

Research and Practice
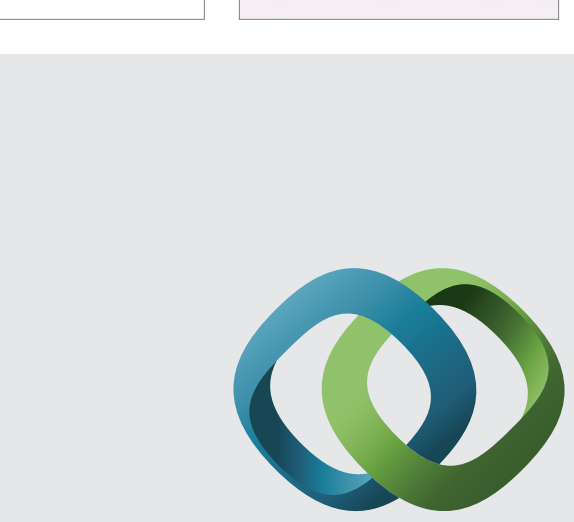

\section{Hindawi}

Submit your manuscripts at

http://www.hindawi.com
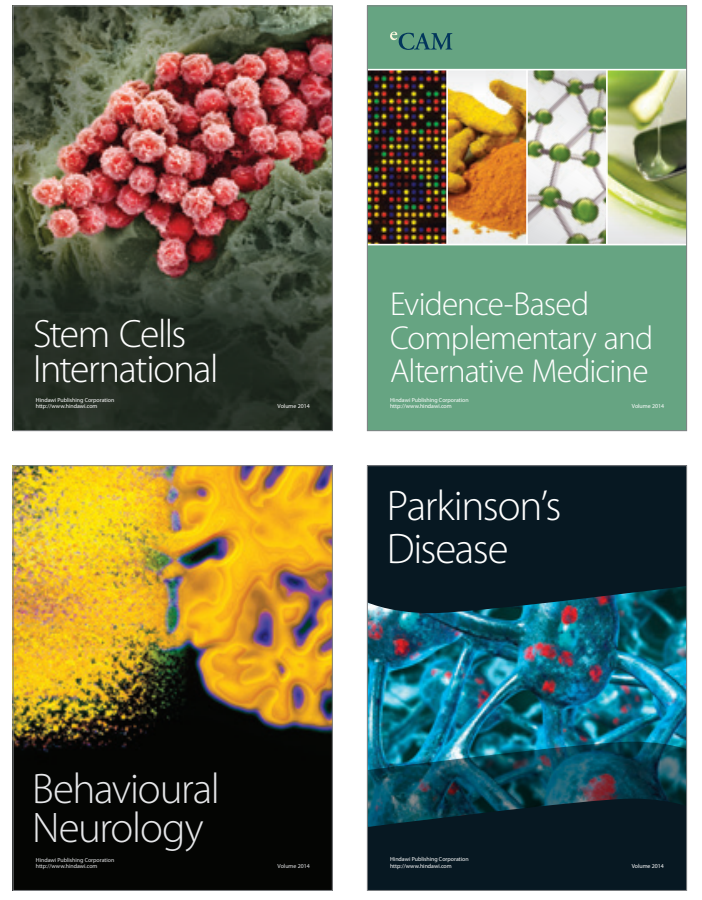
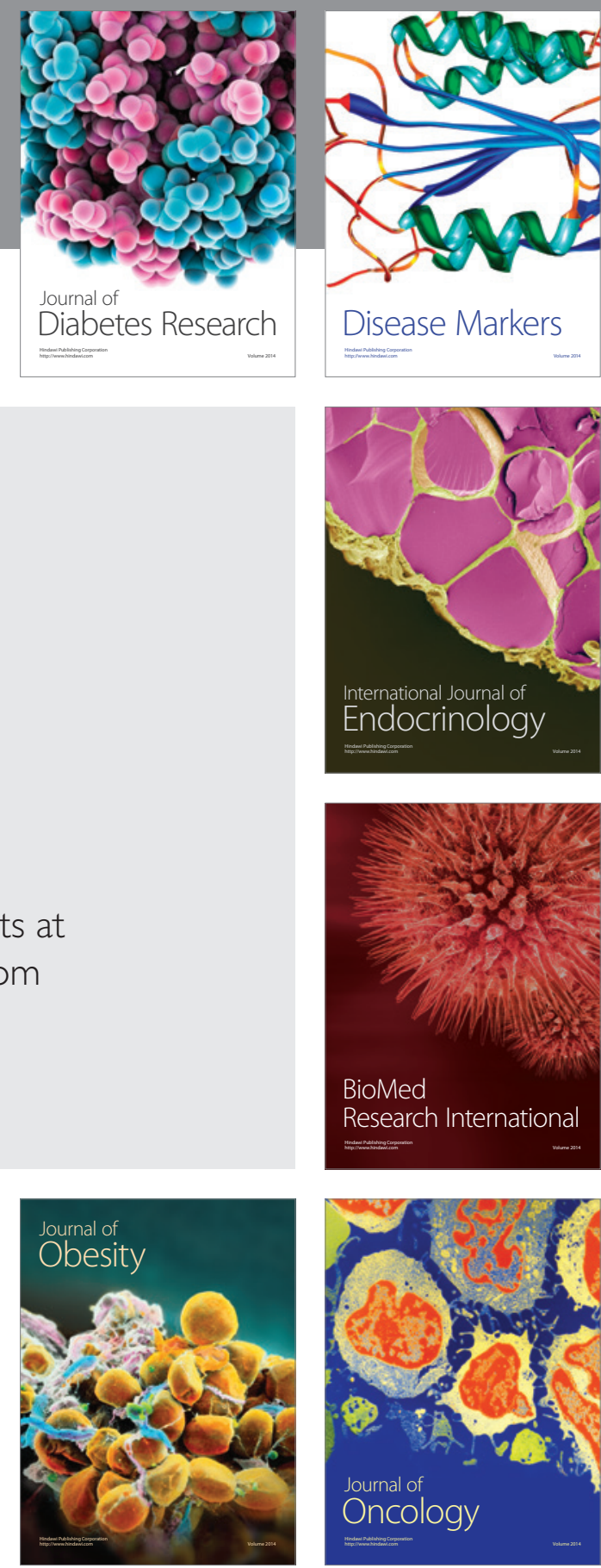

Disease Markers
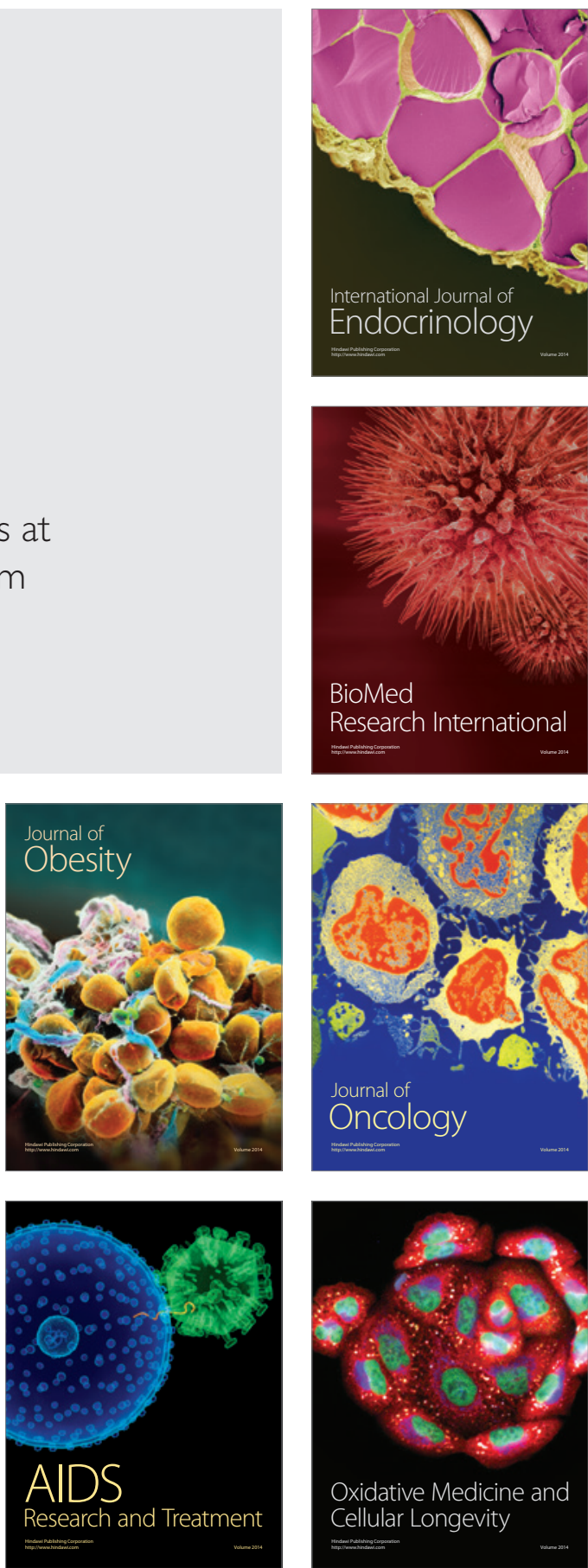